\section{Plate and Frame Filter Presses}

\author{
By E. C. Alford
}

T. SHRIVER AND Co., HAMILTON ST., HARRISON, N. J.

Of the many designs and makes of filtering machines that have been produced, the flush plate and frame, and recessed plate types enjoy the reputation of being the simplest and most efficient machines on the market. Their extreme simplicity of construction and operation is probably the outstanding feature of their superiority. Owing to this simplicity, a filter press is to a large extent independent of the "personal equation" and is as far as possible fool-proof, and cheap unskilled labor can be easily trained to produce satisfactory results.

\section{Description of Press}

A filter press consists of two end supports, rigidly holding two horizontal steel bars. Resting on these side bars are a number of recessed plates or flush plates and frames, the plates having a drainage surface with a machined portion around the outer edge forming a joint surface. The cloths are held between the joint surfaces and act as gaskets in preventing the material that is pumped into the press from leaking out.

One end of the filter press frame holds a screw fitted with a closing device for pressing the recessed plates or the plates and frames together. This squeezing presses the cloth tightly between the machined surfaces and is not for the purpose of pressing the liquid from the solid, as is sometimes believed by the uninformed. When the material to be filtered is pumped into the press it enters a series of clothlined chambers which are "in parallel," that is, each is fed from a common channel and each discharges separately. In the open delivery press they discharge through individual cocks into an open trough; in the closed delivery type they discharge into a header which is piped through the head of the press and connected to a pipe line. The pressure with which the liquid is forced into the filter forces the liquid through the filtering medium. The filtrate flows along the drainage surface of the plates and out, either through the outlet cocks or into the internal closed outlet channel.

During the initial stages of filtration, the pressure rarely goes above 2 or $3 \mathrm{lbs}$. per sq. in. The filter cloth gradually ceases to be the actual filtering medium as the deposit of solids on its surface increases. Therefore, the thicker the deposit or filter bed, the greater is the resistance to the passage of the liquid and the closer the particles are driven together. The filtration pressure gradually increases until a maximum pressure is reached at which efficient filtration ceases.

\section{Washing in the Plate Press}

In its capacity to wash the cake of residue quickly and thoroughly the plate and frame filter excels. The cakes are formed in closed chambers and are therefore solid and supported on all sides, so that there is no possibility of the cake falling from the cloths, resulting in imperfect washing.

Properly formed filter press cakes must of necessity always be uniform throughout. Should there exist soft centers or a settling out of coarser particles, that portion of the cake would be more porous. The flow of the solution, following the line of least resistance, would be of greater volume at such a point than at any other, and a greater amount of solids would be deposited there, until the cake offered a resistance to the flow equal to that of any other portion of the cake.

With the cakes of even permeability formed by the press, high efficiency washing is obtained by admitting the washing liquid back of the cloths. In this way, the wash covers the whole surface of the cloth before penetrating the cake, and all portions of the cake are washed equally.

It must be remembered that efficiency in washing is largely dependent upon the homogeneity and hardness of the cake, which, in turn, is determined by the maximum pressure used during filtration. Therefore, accurate comparative results in washing can be obtained only when the cakes are of equal thickness and hardness. This fact is often overlooked and leads to erroneous conclusions in regard to the washing efficiency of filter presses as compared with other filtering devices. As no air is necessary to hold the cakes in place, there is no possibility of cracks developing in the residue. Such cracks prevent thorough washing.

After the washing operation, air blown through the cakes in the filter press produces a low moisture content.

Filter presses are built to employ positive pressure in filtration.

\section{Recessed Filuter Presses}

In recessed filter presses the cake is formed between two recessed plates, the thicker edge of these plates forming the joint surface and the recess, when two plates are brought together, forming a chamber in which the cake is made. Recessed plate filter presses may be of the open or closed delivery type, and may be either washing or nonwashing. A recessed press, however, is not an efficient washing press, because of the fact that a recessed cake always has a more or less soft core around the feed channel, and the edge of the cake is thinner than the body of the cake. This is the result of the gentle slope from the joint surface to the deepest part of the recess, which is necessary so that the cloth may be gradually stretched to conform to the recess. If the construction were otherwise, the cloth would be stretched so violently that it would break under the pressure at the inside of the joint surface.

The thickness of cakes in a recessed press is limited by the fact that the cloth cannot be stretched too much or its life will be very short and the cloth upkeep would be enormous. In most cases the depth of recess is limited to 0.625 in., making a cake 1.25 in. thick. If for any reason a thicker cake is desired, it is necessary to use spacing frames between the plates or to use a flush plate and frame pattern press.

Recessed plate filter presses are generally of the center feed type, although in some cases the feed inlet may be located in the upper center or in one cormer of the plate.

\section{Plate and Frame Presses}

FIush plate and frame presses may be either washing or nonwashing, and open or closed delivery. The method of feeding plate and frame presses divides them into two general classes: those with internal and those with external feed channels. In the internal feed press, all feed channels are through internal eyes which are cast in the plates and frames inside of the joint surfaces and are connected to each chamber by cored inlet ports in the frames, through which the liquid is admitted. In a washing press, the washing or alternate plates have cored ports connecting the drainage surface of the plates with the washing channel for the admission of wash water back of the cloths. In a closed delivery press, similar ports connecting with the discharge channel are provided in every plate.

The internal feed press requires cloths with holes cut in them to correspond with the holes in the plates. When the cloths are draped over the plates the holes in the cloths should be opposite one another and in direct line with the holes in the plates and frames which form the internal channels. 
In the case of the external feed press, which is the type more commonly used, the feed and washing channels are in external lugs cast integrally with the plates and frames. These lugs are made tight by means of rubber collars. On the feed side the collar is inserted in the plate so that it does not interfere with the inlet port in the frame. On the washing side the collar is inserted in the frame so as not to interfere with the washing ports in the plate. These collars are so made that, when the press is closed, the rubber is pressed against the metal, making tight joints around the external channels. The main advantage of a side feed press is that no holes need be cut in the cloths. The cloths are merely cut long enough so that when draped over the plates they will cover both sides and extend a few inches below the bottom edge of the plates to allow for possible shrinkage. Should the cloths shrink there are no holes to get out of alignment. The cloth should be wide enough to extend beyond the finished joint surfaces of the plates, but not so wide that the edges will reach the finished surface around the holes in the lugs which form the channels.

Another advantage of the flush plate and frame press is that, since there is no recess in the plate, filter paper may be used as well as filter cloth. The manner of applying the paper is first to put the cloth over the plate, and then drape a sheet of filter paper over the cloth. The cloth between the paper and the filtering surface supports the paper. Paper was used formerly considerably in filtrations, but the present procedure is to use a filtration aid such as infusorial earth, which not only is cheaper than paper but produces better results.

In a plate and frame press, frames can be made of any practical thickness.

Flush plate and frame presses are the most efficient washing presses. The cake, being of uniform thickness throughout, offers a uniform resistance to the washing solution, and can therefore be washed free from soluble material with a minimum amount of washing liquid.

\section{Construction and Materials}

Filter presses are usually made of cast iron. Such presses are suitable for neutral or alkaline liquors. Presses for acid liquors or for filtering such materials as colors, pigments, etc., which would be stained or discolored by contact with iron are generally made of wood. For strong sulfuric acid solutions they may be made of hard lead, or for special purposes they may be made of bronze or other metals.

woon-Wooden plates and frames are made of a variety of woods, but, on the whole, resinous woods are considered best, as they have the greatest uniformity of grain and undergo smaller shrinkage and expansion in use. They are also less liable to warp in contact with aqueous solutions. The most commonly used material, and the best for most purposes, is selected yellow pine. The wood used must be free from knots and blemishes of any kind, and the more resinous it is, the better. Wood of small, fine grain is better than wood with a coarse grain. Presses made of this material will stand hydrochloric acid up to 10 per cent and sulfuric acid up to 25 per cent, if used cold. Yellow pine presses will not stand hot solutions, as the hot solution extracts the resin from the wood and makes it weak and spongy. Wooden filter presses are not recommended for hot solutions, but where a hot solution must be filtered in a wood press, maple or oak is the best wood to select. Cypress is very acidresistant but is rather too soft for filter-press use. Too much care cannot be taken in the construction of wooden filter presses, as a poorly made wooden filter press is an item of constant expense, loss, and annoyance. The plates should be made so thick that they do not warp easily, and all joints should be as smooth and perfectly made as possible.

The making of a wooden filter press requires the highest class of wood working skill. Even though the best procurable lumber is purchased, much of this must be discarded in the final inspection. Wooden presses should be set up as soon as made, and kept moist under pressure; otherwise the plates and frames are apt to warp and leak. After a wooden filter press has been used and is put out of service for a time, it should be thoroughly washed with water to remove all mother liquors so that they will not concentrate by evaporation, and attack the wood. The press should then be kept tightly clased and full of water.

In starting up a new wooden press, the tie rods should be loosened until they are only hand-tight, and as the wood expands, the nuts should be backed off so that they will not cut into the wood. A wooden filter press is not tight when made, but becomes tight with swelling. Wooden plates are generally made with corrugated filtering surfaces.

FILTERING SURFACES-There are various types of filtering surfaces: fine grooves, coarse grooves, various sizes of pyramids, double crimped wire cloth, and perforated plates. The most important point is that the channels should be deep enough and narrow enough so that they will not be closed by the cloth being forced into them by the internal pressure. In the second place, the cloth must be supported on a minimum area so as to cut down the amount of nonfiltering surface. The filtering surfaces should be smooth, with no sharp corners or points to cut the cloths. In order of efficiency, the pyramid surface comes first, followed by the corrugated surface, with the perforated screen last. The pyramid surface generally used is about 25 per cent more efficient than any other types of drainage. It holds the cloth away from the surface of the plate and allows free passage of the filtrate through and back of the cloth. At the same time it supports the cloth on numerous points of small area.

In a grooved surface there is much more supported area which is nonfiltering than in the pyramid surface. In the perforated screen the only active area is the cloth over the holes. The relative areas in the generally made types are: pyramid surface 100 , grooved surface 73 , and perforated sereen 30.

CLOSING GEARS-The closing gear most commonly used is a screw and ratchet. This ratchet is provided with a bar of the proper length, and the smaller-sized presses can be closed by one man. In the larger-sized presses, however, a gear and pinion is furnished with a ratio of 4 or $5: 1$. This facilitates tight closing with less exertion, although it takes longer.

Another method commonly used for large installations is the hydraulic closing device, in which the screw end of the press is fitted with a hydraulic ram which operates against the follower and closes the press. With this equipment, which necessitates a hydraulic pump and accumulator, all that is necessary to close the press is to open a valve. In some cases two pressures are used in closing a press; a low pressure which is drawn from the ordinary city main or plant supply closes the press part way. The low pressure line is then closed and the high pressure line opened, and a pressure of from 800 to $2000 \mathrm{lbs}$. is put against the ram. This closes the press tightly, and the valve may then be closed, for the water that is behind the ram will keep the press from opening. In some cases, however, a locking device is employed so that the high pressure may be released.

In opening the press, the mechanical hold-in is released and the release valve opened, whereby a set of cast-iron weights pulls back the ram. The press is then discharged as usual by hand. 